\title{
Correction to: Development of an efficient transformation method by Agrobacterium tumefaciens and high throughput spray assay to identify transgenic plants for woodland strawberry (Fragaria vesca) using NPTII selection
}

\author{
Christopher J. Pantazis ${ }^{1} \cdot$ Sarah Fisk ${ }^{1} \cdot$ Kerri Mills $^{2} \cdot$ Barry S. Flinn $^{1,2,3} \cdot$ Vladimir Shulaev $^{4} \cdot$ Richard E. Veilleux $^{2}$. \\ Yinghui Dan ${ }^{1,2,3}$
}

Published online: 26 February 2022

(c) Springer-Verlag GmbH Germany, part of Springer Nature 2022

\section{Correction to: Plant Cell Rep (2013) 32:329-337 https://doi.org/10.1007/s00299-012-1366-1}

In the Materials and Methods, we stated-in error-that the acetosyrigone added to inoculation medium was $200 \mathrm{mM}$, when it was actually $200 \mu \mathrm{M}$. The error appears again in the Results and Discussion. Also in the Results and discussion is another error about acetosyringone concentration. We stated, "Zhao et al. (2004) reported that an A. tumefaciens density of $2 \times 10^{8} \mathrm{cfu} \mathrm{ml}^{-1}$ in MS liquid medium supplemented with $20 \mathrm{mM}$ acetosyringone (pH 5.4) efficiently produced transgenic octoploid strawberry plants." In reality Zhao et al. used $20 \mu \mathrm{M}$.

The authors apologize for the errors.

Publisher's Note Springer Nature remains neutral with regard to jurisdictional claims in published maps and institutional affiliations.

The original article can be found online at https://doi.org/10.1007/ s00299-012-1366-1.

Yinghui Dan

yinghuidan@aol.com

1 Institute for Advanced Learning and Research, 150 Slayton Avenue Danville, Danville, VA 24540, USA

2 Department of Horticulture, Virginia Polytechnic Institute and State University, Blacksburg, VA 24061, USA

3 Department of Forest Resources and Environmental Conservation, Virginia Polytechnic Institute and State University, Blacksburg, VA 24061, USA

4 Department of Biological Sciences, University of North Texas, Denton, TX, USA 